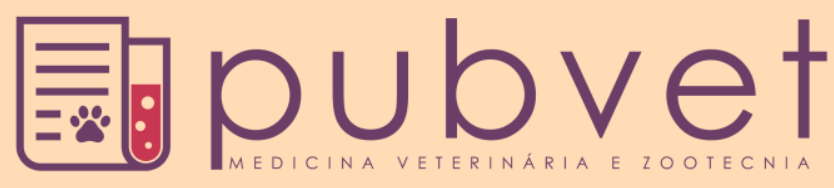

HTTP://DX.DOI.ORG/10.22256/PUBVET.V11N9.940-946

\title{
Desempenho e viabilidade econômica de novilhos em semi confinamento a pasto no Pantanal
}

\author{
Luiz Carlos Pereira ${ }^{1,2 *}$, Álvaro Luiz Pinheiro Carneiro ${ }^{3}$, Luís Carlos Vinhas Ítavo4, \\ Rodrigo Gonçalves Mateus ${ }^{5,1}$, Elizeu Aparecido Messias, ${ }^{2,1}$, Aline Franco da Silva ${ }^{7,1}$, \\ Rafael de Oliveira Lima ${ }^{6,1}$, João Victor de Souza Martins ${ }^{6,1}$, Alysson Martins \\ Wanderley ${ }^{7,1}$
}

${ }^{I}$ Laboratório de Biotecnologia Aplicada a Nutrição Animal - Universidade Católica Dom Bosco - UCDB, Campo Grande MS.

${ }^{2}$ Programa de Pós-graduação em Ciências Ambientais e Sustentabilidade Agropecuária, UCDB, Campo Grande MS. e-mail: luizcp.agro@gmail.com ${ }^{3}$ Medico Veterinário, Fazenda São Geraldo, Bonito, MS, e-mail: alvaroluiz_pinheiro@hotmail.com

${ }^{4}$ Docente do Programa de Pós-graduação em Ciência Animal - Universidade Federal Mato Grosso do Sul - UFMS, Campo Grande MS. e-mail: luis.itavo@ ufms.br ${ }_{5}^{5}$ Docente do Curso de Zootecnia e Medicina Veterinária, Universidade Católica Dom Bosco - UCDB, Campo Grande MS. e-mail: rf4789@ucdb.br

${ }^{6}$ Graduando do Curso de Zootecnia,UCDB.E-mail: eliseumessiasem@gmail.com; rafaelloliveira96@gmail.com; ioaomartins.zootec@gmail.com

${ }^{7}$ Graduando do Curso de Medicina Veterinária, Universidade Católica Dom Bosco - UCDB, Campo Grande MS. e-mail: alinemsfranco@gmail.com

${ }^{7}$ Doutorando em Ciência Animal, Universidade Federal do Mato Grosso do Sul-UFMS, Campo Grande MS. e-mail: alyssonmw@yahoo.com.br

RESUMO. O objetivo deste trabalho foi avaliar o desempenho de dois grupamentos genéticos de novilhos F1 Angus*Nelore e Nelore em confinamento a pasto e sua viabilidade econômica. O experimento foi conduzido na Fazenda São Geraldo localizado no município de Bonito - MS. Foram utilizados 94 novilhos, sendo distribuídos em blocos casualizado, totalizando dois tratamentos. Tratamento $1-50$ novilhos F1 Angus x Nelore peso médio de 459,32 kg, Tratamento 2 - 44 novilhos Nelore com peso médio de 454,45 $\mathrm{Kg}$. Analise de desempenho dos animais foi significativa para efeito $(\mathrm{P}<0,05)$, entre as raças de ganho de peso final, ganho de peso médio total, ganho de peso médio diário e rendimento de carcaça. Os animais $\mathrm{F} 1$ apresentaram maior ganho de peso médio total com $125 \mathrm{~kg}$ e o Nelore com $120 \mathrm{~kg}$, representando um ganho de $5 \mathrm{Kg}$ superior. O ganho de peso médio diário para o cruzamento F1 foi 1,53 kg e o Nelore apresentou 1,28 kg, observando um desempenho de 22,3 \% superior do F1 em relação ao Nelore. A análise econômica demonstrou efeito para tratamento $(\mathrm{P}<0,05)$ para os custos operacionais $(\mathrm{CO})$, receita operacional total (ROT), custo de capital (CK), margem bruta, margem liquida, resultado econômico, ponto de equilíbrio, margem liquida por $\mathrm{kg}$ de peso vivo. A lucratividade dos animais $\mathrm{F} 1 \mathrm{com} 14,74 \%$ e uma rentabilidade de $17,59 \%$, enquanto os animais Nelores ficaram com 2,04\% de lucratividade inferior e uma rentabilidade $14,86 \%$. Conclui-se que o cruzamento entre animais Nelore e Angus com suplementação obteve maior desempenho de peso e rentabilidade.

Palavras chaves: Bovinos, suplementação a pasto, cruzamento industrial, nutrição

\section{Performance and economic viability of finishing of steers in pasture supplemented in the Pantanal}

ABSTRACT. The objective of this study was to evaluate the performance of two genetic groups of F1 steers Angus vs. Nellore and Nellore in pasture supplementation and their economic viability. The experiment was conducted at the São Geraldo farm located in the municipality of Bonito - MS, Brazil. It was used 94 steers, being distributed in randomized blocks, totaling two treatments. Treatment $1-50$ Angus vs. Nellore F1 steers weight 459.32, Treatment $2-44$ Nellore steers with average weight of $454.45 \mathrm{~kg}$. Performance analysis of the animals was significant $(\mathrm{P}<0.05)$, between the genetic groups of final weight gain, total average weight gain, average daily weight gain and carcass dressing. The 
F1 animals had the higher total weight gain with $125 \mathrm{~kg}$ and the Nellore with $120 \mathrm{~kg}$, representing a gain of $5 \mathrm{~kg}$ higher. The daily average weight gain for F1 crossing was 1.53 $\mathrm{kg}$ and Nellore presented $1.28 \mathrm{~kg}$, observing a performance of $22.3 \%$ higher than F1 in relation to Nellore. The economic analysis showed a treatment effect $(\mathrm{P}<0.05)$ for operating costs $(\mathrm{CO})$, total operating revenue (ROT), cost of capital (CK), gross margin, net margin, economic break-even point, margin Per kg live weight. The profitability of F1 animals with $14.7 \%$ and a profitability of $17.6 \%$; while the Nellore animals were with $2.0 \%$ lower profitability and a profitability of $14.9 \%$. It was concluded that the cross between Nellore and Angus animals with supplementation obtained greater performance of weight and profitability

Keywords: Cattle, pasture supplementation, industrial crossing, nutrition

\section{Desempeño y viabilidad económica de novillos semiestabulados a pasto en el Pantanal}

RESUMO. El objetivo de este trabajo fue evaluar el desempeño de dos grupos genéticos de novillos F1 Angus * Nelore y Nelore estabulados a pasto y su viabilidad económica. El experimento fue conducido en la Hacienda São Geraldo ubicado en el municipio de Bonito - MS, Brasil. Se utilizaron 94 bovinos, siendo distribuidos en bloques casualizados, totalizando dos tratamientos. Tratamiento 1 - 50 becerros F1 Angus x Nelore peso medio de 459,32 kg, Tratamiento 2 - 44 novillos Nelore con peso medio de 454,45 Kg. El análisis de rendimiento de los animales fue significativo para el efecto $(\mathrm{P}<0,05)$, entre las razas de ganancia de peso final, la ganancia de peso medio total, la ganancia de peso diario y el rendimiento de la carcasa. Los animales $\mathrm{F} 1$ presentaron una mayor ganancia de peso medio total con $125 \mathrm{~kg}$ y el Nelore con $120 \mathrm{~kg}$, representando una ganancia de $5 \mathrm{Kg}$ superior. La ganancia de peso promedio diario para el cruce F1 fue de 1,53 kg y el Nelore presentó 1,28 $\mathrm{kg}$, observando un desempeño del 22,3\% superior del F1 en relación al Nelore. El análisis económico demostró efecto para el tratamiento $(\mathrm{P}<0,05)$ para los costos operativos $(\mathrm{CO})$, ingreso operativo total (IOT), costo de capital (CK), margen bruto, margen neto, resultado económico, punto de equilibrio, margen por kg de peso vivo. La lucrativita de los animales F1 con el 14,74\% y una rentabilidad del 17,59\%, mientras que los animales Nelores quedaron con un $2,04 \%$ de rentabilidad inferior y una rentabilidad $14,86 \%$. Se concluye que el cruce entre animales Nelore y Angus con suplementación obtuvo mayor desempeño de peso y rentabilidad.

Palabras claves: Bovinos, cruce industrial, nutrición, suplementación a pasto

\section{Introdução}

O Brasil é considerado o maior exportador mundial de carne bovina, produzindo um índice de abate de 43,3 milhões de animais por ano e, para isso possui o maior rebanho comercial do mundo, com aproximadamente 200 milhões de cabeça (ANUALPEC, 2016, FAPRI, 2016). No cenário do agronegócio brasileiro o mesmo gera um faturamento de mais de $\mathrm{R} \$ 50$ bilhões ano, oferecendo cerca de 7,5 milhões de empregos.

Em pastagens tropicais, os bovinos dificilmente conseguem obter das forrageiras todos os nutrientes essenciais a um bom desempenho produtivo (Moreira and Prado, 2010, Moreira et al., 2004). Para Rotta et al. (2010) o fornecimento de suplementação a pasto é uma estratégia eficiente para garantir o desempenho produtivo dos animais. Para alto desempenho e reduzida idade de abate, o pasto deve proporcionar oferta de forragem para seleção de dieta, constituída, sobretudo por folhas (Frescura et al., $\underline{2005) \text {. }}$

Visando o avanço da produtividade e competitividade na bovinocultura, diversas alternativas tecnológicas têm sido propostas e, dentre elas, a utilização de confinamento a pasto (El-Memari Neto et al., 2003, Prado and Moreira, 2002). Esta dieta possui grande versatilidade devida a redução capital imobilizado com maquinas e equipamentos. Segundo Ítavo et al. (2005) a compra ou confecção do concentrado constitui a maior parcela de gastos no sistema de produção de bovinos. A tomada de decisão poderá ser baseada na análise de custos com o intuito de 
obter resultados satisfatórios e rentabilidade na atividade (Barros et al., 2009).

$\mathrm{O}$ desafio a ser vencido pela gestão agropecuária competitiva e a determinação dos custos de produção de bovinos como processo fundamental a tomada de decisão (Prado and Souza, 2009). É mediante resultados econômicos que o produtor pode tomar, conscientemente, suas decisões e encarar o seu sistema de produção de gado de corte como uma empresa rentável (Prado, $\underline{2010}$ ).

Neste contexto este trabalho objetivou avaliar o desempenho de dois grupamentos genéticos de novilhos F1 Angus vs. Nelore e Nelore suplementados a pasto e sua viabilidade econômica.

\section{Material e Métodos}

O experimento foi conduzido na Fazenda São Geraldo, localizada no município de Bonito - MS. $\mathrm{O}$ delineamento experimento utilizado foi inteiramente casualizado com dois tratamentos considerando animais de grupamentos genéticos distintos, respeitando os princípios básicos da experimentação: repetição, casualização e controle local (Ferreira, 2011). Foram utilizados 94 novilhos, sendo distribuídos em dois lotes descritos abaixo:

Tratamento $1-50$ novilhos F1 Angus x Nelore peso médio de $459,32 \pm 14.35 \mathrm{~kg}$;

Tratamento $2-44$ novilhos Nelore com peso médio de $454,45 \pm 18.85 \mathrm{~kg}$.

Todos os animais foram previamente pesados, identificados, vermifugados e submetidos ao controle de ectoparasitos antes do início do experimento. Durante o período experimental foram realizadas pesagens mensais, sempre no período matutino.

Os grupos foram alojados em um piquete com áreas de $10 \mathrm{ha}^{-1}$ para tratamento 1 e lotação media 5,1 unidades animal (UA) e o tratamento 2 as áreas foram de 9,35 ha ${ }^{-1}$ com lotação média de 4,75 UA, em sistema de suplementação a pasto de Urochloa brizantha cv. Xaraés, com fornecimento de água ad libitum.

As dietas foram constituídas milho grão seco moído, caroço de algodão e núcleo proteico contendo: farelo de soja, ureia, núcleo mineralvitamínico e aditivo promotor de crescimento (Tabela 1).
Tabela 1. Teores bromatológicos de concentrado comercial para bovinos

\begin{tabular}{lc}
\hline Variáveis & Concentrado, \% \\
\hline Matéria seca & 87,45 \\
PB & 18,00 \\
Fibra em detergente neutro & 19,35 \\
Extrato etéreo & 2,4 \\
Matéria mineral* & 7 \\
Saccharomyces cerevisiae** & - \\
Nutrientes digestíveis Totais & 74,35 \\
*Composição centesimal do concentrado foi: cálcio 1,35\%, fósforo \\
0,5\%, magnésio 0,05, enxofre 0,1065\%, sódio 0,7\%, cobalto 0,3\%, \\
selênio 0,081\%, iodo 0,4\%, zinco 1,8\%, manganês 5.46\%, cobre 3\%, \\
**S.s cerevisiae 3,0000x10 E7 UFC/kg. Concentrado comercial para \\
bovinos - União Nutrição Animal.
\end{tabular}

Os alimentos foram fornecidos à vontade, por voltas das 16:00 horas, sendo que as sobras foram registradas e pesadas diariamente para a determinação do consumo diário. A quantidade de ração fornecida foi calculada de modo a permitir aproximadamente 5\% de sobras.

Foi avaliado o desempenho de ganho de peso dos animais durante o período experimental, onde que os animais do tratamento 1 iniciando no dia $31 / 07 / 2015$ e finalizado no dia $19 / 10 / 2015$; os animais do tratamento 2 iniciando no dia $15 / 07 / 2015$ e finalizado no dia 19/10/2015.

As pesagens dos animais foram realizadas no início do experimento e no final do experimento, após jejum de sólidos de 12 horas. Ao final da pesquisa foram calculados os índices de desempenho de ganho de peso médio diário (GMD), ganho de peso médio total (GMT) e rendimento de carcaça pós morten.

A metodologia utilizada neste estudo para cálculo de custos de produção foi realizada por meio da divisão de custo operacional efetivo (COE), custo operacional total (COT) e custo total (CT), conforme Ferreira et al. (2004). A classificação dos custos permite identificar os seguintes indicadores de resultado: receita total (RT), Custo total (CT), lucro operacional (LO) e margem líquida (ML). A lucratividade resulta da ML dividida pela RT. A rentabilidade resulta da ML dividida pelo investimento total (Martin et al., 1998). A margem por $\mathrm{kg}$ de novilho para abate ou de carcaça foi obtida ao subtrair do valor de RT o $\mathrm{CT} / \mathrm{kg}$ de novilho para abate ou carcaça, e esse valor foi dividido pela quantidade produzida de novilho. A determinação do ponto de equilíbrio foi calculada pela seguinte equação: $\mathrm{Q}=\mathrm{CF} / \mathrm{P}-\mathrm{CVu}$. Em que: Q é a quantidade de carne produzida, em $\mathrm{kg}$, CF é o custo fixo total, $\mathrm{P}$ é o preço de mercado 
do produto $(\mathrm{R} \$ / \mathrm{kg})$ e Cvu é o custo variável unitário.

Os dados foram submetidos à análise estatística segundo o procedimento PROC GLM do pacote estatístico SAS (2004) versão 9.1.. Para a comparação das médias, as variáveis foram submetidas ao teste de Mann-Whitney $(\mathrm{P}<0,05)$.

\section{Resultados e discussão}

Análise de desempenho dos novilhos apresentou efeito $(\mathrm{P}<0,05)$, para ganho de peso final, ganho de peso médio total, ganho de peso médio diário e rendimento de carcaça (Tabela 2).

Tabela 2. Desempenho de novilhos terminados em confinamento a pasto e recebendo $10 \mathrm{~kg}_{\text {.dia }}{ }^{-1} \mathrm{de}$ concentrado

\begin{tabular}{|c|c|c|c|c|c|}
\hline \multirow{2}{*}{ Variáveis } & \multirow[b]{2}{*}{$\mathrm{n}$} & Angus*Nelore & Nelore & \multirow{2}{*}{$\mathrm{CV}$} & \multirow{2}{*}{$\mathrm{P}$} \\
\hline & & 50 & 44 & & \\
\hline Peso Inicial, kg & & $459 \mathrm{a}$ & $454 \mathrm{a}$ & 15,79 & 0,9261 \\
\hline Peso Final, kg & & $584 \mathrm{a}$ & $574 \mathrm{~b}$ & 15,08 & 0,0001 \\
\hline Ganho de Peso Médio Total, kg & & $125 \mathrm{a}$ & $120 b$ & 15,34 & 0,0001 \\
\hline Ganho de Peso Médio Diário, kg & & $1,56 \mathrm{a}$ & $1,28 b$ & 15,34 & 0,0001 \\
\hline Peso de carcaça, kg & & $321,2 \mathrm{a}$ & $321,5 \mathrm{a}$ & 23,38 & 0,8751 \\
\hline Rendimento de carcaça, $\%$ & & $55,0 \mathrm{~b}$ & $56,0 \mathrm{a}$ & 15,65 & 0,0001 \\
\hline
\end{tabular}

Coeficiente de variação $(C V)$; Efeito do Tratamento $(P)$; Médias da mesma linha com letras minúsculas diferentes foram significativa pelo teste Mann-Whitney $(\mathrm{P}<0,05)$.

Os novilhos F1 obtiveram $10 \mathrm{~kg}$ a mais de peso final em relação ao tratamento 2 que apresentou $574 \mathrm{~kg}$ com 94 dias de suplementação, ou seja, com um período de 14 dias a mais que os novilhos F1. Os desempenhos de peso final obtidos no presente estudo são relatados por outros autores, denotando que os novilhos superioridade dos F1 na terminação para o abate em relação ao Nelore (Ítavo et al., 2008).

Em relação ao ganho médio total os novilhos F1 apresentaram o maior valor, $125 \mathrm{~kg}$ e o Nelore com $120 \mathrm{~kg}$, representando um ganho de $5 \mathrm{~kg}$ superior. Nota-se entre os tratamentos um desempenho de GMD de 22,3 \% superior do F1 em relação ao Nelore que apresentou GMD de $1,28 \mathrm{~kg}$ (Tabela 2). Oliveira et al. (2011) relataram desempenhos semelhante para animais F1 angus com ganhos de $1,54 \mathrm{~kg}$ dia. No entanto, Marcondes et al. (2011) ressalvam que apesar do menor desempenho dos animais Nelore, o ganho médio diário da raça demonstra grande potencial.

Entretanto, o Nelore apresentou maior rendimento de carcaça após o abate com $56 \%$, enquanto o F1 apresentou 55\%. Em referência às relações com peso de corpo vazio, Owens et al. (1993) afirmaram que raças menores possuem maior percentual de órgãos e vísceras e gordura interna, o que não foi observado, possivelmente devido aos efeitos do cruzamento. A análise do centro de custo verificou efeito $(\mathrm{P}<0,05)$ para juros de capital e juros de capital de giro. Sendo os novilhos Nelores obtiveram custos com juros de capital 14,0\% maiores que os novilhos F1 devido à alimentação que representou o maior custo operacional auferido no período experimental, representando uma diferença no valor de $\mathrm{R} \$$ 5.922,00 entre os lotes analisados. O mesmo fato que influenciou diretamente sobre os juros de capital de giro que apresentou um aumento de $\mathrm{R} \$$ 3,00 para cada novilho Nelore em relação aos F1 (Tabela 3).

A análise de viabilidade econômica demonstrou efeito $(\mathrm{P}<0,05)$ para custo operacional (CO), receita operacional total (ROT), custo de capital (CK), margem bruta, margem liquida, resultado econômico, ponto de equilíbrio, lucratividade, rentabilidade e margem liquida por $\mathrm{kg}$ de peso vivo (Tabela 4). Não houve efeito para os demais fatores de viabilidade econômica avaliados durante o período experimental.

O custo operacional efetivo os animais Nelore foi $\mathrm{R} \$ 477,18$, os valores foram 7,3\% superiores ao F1. Já o custo de capital verificou um gasto de $18,1 \%$ superior do Nelore em relação ao F1. Tal fato está associado ao maior período de terminação impetrado ao investimento do animal e o capital de giro do sistema de produção.

A receita operacional total os novilhos $\mathrm{F} 1$ foram mais representativos com valor $\mathrm{R} \$ 642,03 \mathrm{e}$ os novilhos Nelore $\mathrm{R} \$ 627,67$, impactando na sua margem bruta que obteve uma redução de ganho financeiro de $\mathrm{R} \$ 74,20$. Da mesma forma a 
margem liquida com redução de $10,61 \%$ do Nelore em relação ao F1. Ítavo et al. (2008) observaram entre os tratamentos e os grupos genéticos, considerando-se essa diferença na margem líquida de grande importância para o produtor, principalmente nos sistemas de ciclo completo.

Tabela 3. Centro de custo de garrotes em terminação em confinamento a pasto durante o período experimental

\begin{tabular}{lcccc}
\hline Variáveis & $\mathrm{F} 1$ & Nelore & $\mathrm{CV}$ & $\mathrm{P}$ \\
\hline Período de confinamento & 80 & 94 & - & - \\
Preço kg vivo compra, R\$ & 9,02 & 9,02 & - & - \\
Preço kg vivo venda, R\$ & 9,34 & 9,34 & - & - \\
Preço do Suplemento, R\$ & 0,45 & 0,45 & - & - \\
Custo do Suplemento, dia ${ }^{-1}$ & 4,50 & 4,50 & - & - \\
Suplementação, R\$/animal/período & 360,00 & 423,00 & - & - \\
Sanidade, R\$/animal/período & 2,48 & 2,48 & - & - \\
Manutenção, R\$/animal/período & 80,00 & 94,00 & - & - \\
K Investido Animal, R\$ & $2.070,09 \mathrm{a}$ & $2.047,54 \mathrm{a}$ & 15,25 & 0,1901 \\
Juros k, R\$ & $36,99 \mathrm{a}$ & $42,98 \mathrm{~b}$ & 13,38 & 0,0001 \\
Juros k giro, R\$/animal/período & $7,91 \mathrm{a}$ & $10,91 \mathrm{~b}$ & 31,42 & 0,0001 \\
\hline
\end{tabular}

Coeficiente de variação $(C V)$; Efeito do Tratamento $(P)$; Médias da mesma linha com letras minúsculas diferentes foram significativa pelo teste Mann-Whitney $(\mathrm{P}<0,05)$. Fonte: Preço e cotação $\underline{\text { Scot Consultoria (2015). }}$

Tabela 4. Viabilidade econômica de garrotes em terminação no confinamento a pasto durante período experimental

\begin{tabular}{|c|c|c|c|c|}
\hline Variáveis & $\mathrm{F} 1$ & Nelore & $\mathrm{CV}$ & $\mathrm{P}$ \\
\hline Custo operacional efetivo, $\mathrm{R} \$$ animal $^{-1}$ & $442,48 \mathrm{a}$ & $519,48 b$ & 1,23 & 0,0001 \\
\hline Custo operacional total, $\mathrm{R} \$$ animal $^{-1}$ & $2.512,57 \mathrm{a}$ & $2.567,02 \mathrm{a}$ & 7,14 & 0,3211 \\
\hline Custo de capital, $\mathrm{R} \$$ & $44,89 \mathrm{a}$ & $53,89 \mathrm{~b}$ & 0,57 & 0,0015 \\
\hline 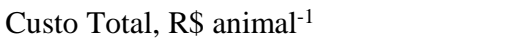 & $2.557,46 \mathrm{a}$ & $2.620,91 \mathrm{a}$ & 0,57 & 0,1755 \\
\hline Receita Operacional Total, $\mathrm{R} \$$ animal $^{-1}$ & $642,03 \mathrm{a}$ & $627,67 \mathrm{~b}$ & 15,82 & 0,0500 \\
\hline Receita Total, $\mathrm{R} \$$ animal $^{-1}$ & $2.999,54 \mathrm{a}$ & $3.002,34 \mathrm{a}$ & 5,34 & 0,0034 \\
\hline Margem Bruta, $\mathrm{R} \$$ animal $^{-1}$ & $2.557,06 \mathrm{a}$ & $2.482,86 b$ & 5,34 & 0,0034 \\
\hline Margem Líquida, $\mathrm{R} \$$ animal $^{-1}$ & $486,97 \mathrm{a}$ & $435,32 b$ & 17,02 & 0,0500 \\
\hline Resultado econômico, $\mathrm{R} \$$ animal $^{-1}$ & $442,08 \mathrm{a}$ & $381,43 b$ & 21,37 & 0,0001 \\
\hline Produtividade média por animal, PRV & 24,00 & 25,02 & - & - \\
\hline Ponto de equilíbrio do peso animal, $\mathrm{kg}$ & $538 \mathrm{a}$ & $550 \mathrm{~b}$ & 7,21 & 0,0015 \\
\hline Preço de nivelamento, $\mathrm{kg}$ & $7,96 \mathrm{a}$ & $8,15 \mathrm{a}$ & 7,41 & 0,0632 \\
\hline Lucratividade, $\%$ & $14,74 \% \mathrm{a}$ & $12,70 \% \mathrm{~b}$ & 16,72 & 0,0012 \\
\hline Rentabilidade, $\%$ & $17,59 \% \mathrm{a}$ & $14,86 \% \mathrm{~b}$ & 19,63 & 0,0450 \\
\hline Margem Líquida, $\mathrm{R} \$ / \mathrm{kg} /$ ganho & $3,54 \mathrm{a}$ & $3,18 \mathrm{~b}$ & 18,36 & 0,0020 \\
\hline
\end{tabular}

Coeficiente de variação $(C V)$; Efeito do Tratamento $(P)$; Médias da mesma linha com letras minúsculas diferentes foram significativa pelo teste Mann-Whitney $(\mathrm{P}<0,05)$.

Dessa forma ao se avaliar o resultado econômico dos sistemas de produção a utilização de animais F1 (R\$ 442,10) demonstrou ganhos financeiros superiores em relação ao Nelore ( $\mathrm{R}$ \$ 381,40). O ponto de equilíbrio entre os tratamentos apresentou o maior custo operacional para o Nelore que obteve o valor de $550 \mathrm{~kg}$, implicando $2,2 \%$ a mais no custo operacional em relação ao F1 (Tabela 4).

Ao se avaliar a margem líquida por $\mathrm{kg}$ ganho os animais F1 apresentou a melhor margem com $\mathrm{R} \$ 3,54$ e os animais Nelore com $\mathrm{R} \$ 3,18$.
Portanto a maior lucratividade $(14,7 \%)$ e rentabilidade $(17,6 \%)$ dos novilhos $F 1$ representaram um ganho de $2,0 \%$ na lucratividade e $2,7 \%$ na rentabilidade. Vale ressaltar que o desempenho econômico é de fundamental importância para a escolha da raça ou do cruzamento a se explorar e, embora seja dependente do desempenho zootécnico, a correlação entre ambos nem sempre é perfeita, pois o resultado de determinado cruzamento pode proporcionar maior produção que outro, porém a custo mais elevado (Guimarães et al., 2005). 
Dessa forma, a definição dos objetivos da suplementação consiste em estratégias de suplementação que devem possibilitar ganho de peso satisfatório e viabilidade econômica. A composição dos custos de produção para terminação.

\section{Conclusão}

O cruzamento entre animais Nelore e Angus com suplementação obteve maior desempenho de peso e rentabilidade.

\section{Referências Bibliográficas}

ANUALPEC. 2016. Anuário da Pecuária Brasileira, 20th edn. Instituto FNP, São Paulo, SP, Brasil.

Barros, C. S., Monteiro, A. L. G., Candal, C. H. E., Poli, J. R. D., Canziani, J. R. F. \& Machado, M. A. 2009. Rentabilidade da produção de ovinos de corte em pastagem e em confinamento1. Revista Brasileira de Zootecnia, 38, 2270-2279.

El-Memari Neto, A. C., Zeoula, L. M., Cecato, U., Prado, I. N., Caldas Neto, S. F., Kazama, R. \& Oliveira, F. C. L. 2003. Suplementação de novilhos nelore em pastejo de Brachiaria brizantha com diferentes níveis e fontes de concentrado. Revista Brasileira de Zootecnia, 32, 1945-1955.

FAPRI. 2016. Food and Agricultural Policy Research Institute. In: Database, W. A. O. (ed.) Food and Agricultural Policy Research Institute. Iowa State University and University of Missouri-Columbia Ames, IA, USA.

Ferreira, D. F. 2011. SISVAR: A Computer Statistical Analysis System. Ciência $e$ Agrotecnologia, 35, 1039-1042.

Ferreira, I. C., Silva, M. A., Reis, R. P., Euclides Filho, K. \& Figueiredo, G. R. 2004. Análise de custos de diferentes grupos genéticos de bovinos de corte terminados em confinamento. Arquivo Brasileiro de Medicina Veterinaria e Zootecnia, 56, 385-391.

Frescura, R. B. M., Pires, C. C., Rocha, M. G., Silva, J. H. S. \& Müller, L. 2005. Sistemas de alimentação na produção de cordeiros para abate aos $28 \mathrm{~kg}$. Revista Brasileira de Zootecnia, 34, 1267-1277.

Guimarães, P. H. S., Madalena, F. E. \& Cezar, I. M. 2005. Simulação dos efeitos dos preços de produtos e insumos na avaliação econômica de três sistemas alternativos de bovinocultura de cria. Arquivo Brasileiro de Medicina Veterinária e Zootecnia, 57, 227-230.

Ítavo, C. C. B. F., Ítavo, L. C. V., Dias, A. M., Silva, J. P. B., Euclides Filho, K. \& Graça, M. M. 2005. Fatores inerentes à economicidade de sistemas de produção de bovinos de corte, no município de Campo Grande, Mato Grosso do Sul. Revista Internacional de Desenvolvimento Local, 6, 119-136.

Ítavo, L. C. V., Tolentino, T. C. P., Ítavo, C. C. B. F., Gomes, R. C., Dias, A. M. \& Silva, F. F. 2008. Consumo, desempenho e parâmetros econômicos de novilhos Nelore e F1 Brangus $\mathrm{x}$ Nelore terminados em pastagens, suplementados com mistura mineral e sal nitrogenado com ureia ou amireia. Arquivo Brasileiro de Medicina Veterinária e Zootecnia, 60, 419-427.

Marcondes, M. I., Valadares Filho, S. C., Oliveira, I. M., Paulino, P. V. R., Valadares, R. F. D. \& Detmann, E. 2011. Eficiência alimentar de bovinos puros e mestiços recebendo alto ou baixo nível de concentrado. Revista Brasileira de Zootecnia, 40, 1313-1324.

Martin, N. B., Serra, R., Oliveira, M. D. M., Ângelo, J. A. \& Okawa, H. 1998. Sistema integrado de custos agropecuáriosCUSTAGRI. Informações Econômicas, 28, 728.

Moreira, F. B. \& Prado, I. N. 2010. Sazonalidade na produção e qualidade de plantas forrageiras. In: Prado, I. N. (ed.) Produção de bovinos de corte e qualidade da carne. Eduem, Maringá, Paraná, Brasil.

Moreira, F. B., Prado, I. N., Cecato, U., Wada, F. Y. \& Mizubuti, I. Y. 2004. Forage evaluation, chemical composition, and in vitro digestibility of continuously grazed star grass. Animal Feed Science and Technology, 113, 239-249.

Oliveira, I. M., Paulino, P. V. R., Valadares Filho, S. C., Detmann, E., Paulino, M. F., Gomide, L. A. d. M., Duarte, M. S. \& Couto, V. R. M. 2011. Carcass traits of Nellore, F1 SimmentalX Nellore and F1 Angus $x$ Nellore steers fed at maintenance or ad libitum with two concentrate levels in the diet. Revista Brasileira de Zootecnia, 40, 2938-2946.

Owens, F. N., Dubeski, P. \& Hanson, C. F. 1993. Factors that alter the growth and development of ruminants. Journal of Animal Science, 71, 3138-3150. 
Prado, I. N. 2010. Produção de bovinos de corte e qualidade da carne. Eduem, Maringá, Paraná, Brasil.

Prado, I. N. \& Moreira, F. B. 2002. Suplementação de bovinos no pasto $e$ alimentos usados na bovinocultura. Eduem, Maringá, Paraná, Brasil.

Prado, I. N. \& Souza, J. P. 2009. Cadeias produtivas: estudo sobre a competitividade e coordenação. Eduem, Maringá, Paraná, Brasil.

Rotta, P. P., Prado, R. M., Moreira, F. B. \& Prado, I. N. 2010. Suplementação do perído do verão. In: Prado, I. N. (ed.) Produção de bovinos de corte e qualidade da carne. Eduem, Maringá, Paraná, Brasil.
SAS. 2004. SAS/STAT. User's Guide: version 9.1. North Caroline, SAS Institute, 5136p.

Scot Consultoria. 2015. Disponível em: https://www.scotconsultoria.com.br. Acesso em: 08 de nov. 2016.

\section{Article History:}

Received 5 April 2017

Accepted 16 May 2017

Available online 9 August 2017

License information: This is an open-access article distributed under the terms of the Creative Commons Attribution License 4.0, which permits unrestricted use, distribution, and reproduction in any medium, provided the original work is properly cited. 